\title{
3D Image and Sound Control Method for User-Perceived 3D Feeling and Functional Game Optimization
}

\author{
Jeong-Hoon Shin ${ }^{1}$ and Seong-Jun Lee ${ }^{2}$ \\ School of Information Technology Eng, Catholic University of Dae-Gu, Korea \\ only4you@cu.ac.kr,lll4444@cu.ac.kr
}

\begin{abstract}
Functional games are games that combine play and study together, and this genre is consolidating its position as a new alternative that has the potential to transform the industry. Functional games are an excellent tool for attaining specific gools such as increasing concentration levels and learning abilities by capitalizing on the entertainment nature of games. Moreover, the scope of use for functional games is beeoming increasingly diverse, ranging from specialized domains such as medical support, medical learning, military simulation, health, special education, and learning tools for everyday education and well being.

To optimize 3D multi-dimensional functional games' benefits, this paper presents an analysis of the level of $3 D$ feel perceived bycusers regarding $3 D$ depth level, distance and location. Moreover, to ensure the optimization of the user's concentration levels and engagement with the games themselves, this paper proposes a system that provides sound effects that incorporate ultrasonic waves' auxiliary sounds to be integrated with traditional sound effects.

When the recommendations of this paper are implemented, it is possible to select the distance and the location than are optimal for viewing in 3D, and it is hypothesized that optimization of user benefits concerning functional games will be enabled through auxiliary sound effects.
\end{abstract}

Keywords: Image control spstem, three-dimensional effect start, Ultrasound, Ultrasound Speaker System

\section{Introduction}

The emergence of $3 \mathrm{D}$ images these days following digital technology advancement has significantly transformed not only the film industry, but also the general public's TV viewing environment as well. In particular, as new 3D contents are emerging, the scope of use for multi-dimens onal 3D images is expanding to a myriad of industries, such as broadcasting, communications, entertainment, education and others [1, 2]. Nicholas Negroponte has explained the benefits thusly; "multi-dimensional 3D images provide the sense of reality as if one is in that particular place; has precise and superb reenactment ability; a powerful effect of grabbing one's attention and the ability to provide images that are more real than reality itself" [3]. By using these 3D image benefits, functional games are trying to convert 2D images to 3D images for functional benefit optimization. Functional games are games that use functional contents as a medium so that training can take place, to realize specific goals such as concentration level increase $[4,5,6]$. These functional games are used mostly for education and therapy, and for other purposes such as training and rehabilitation. Functional 
games' foremost strength is to command the user's full attention while maximizing enjoyment. Moreover, the educational component is also emphasized in a natural, unobtrusive way, by leveraging the fun factor so that the younger generation, who are quite used to video and computer games, can be passionate about it [7].

To analyze the optimal environment for viewing multi-dimensional 3D images, this paper analyzed the level of user perception by measuring the 3D depth level, viewing distance and location for functional optimization. Moreover, ultrasonic waves were used as auxiliary sound when providing games' sound effects to help increase concentration levels when engaged in functional games. To ensure objective comparative analysis, the following three aspects were observed: 2D functional game contents, multi-dimensional 3D functional contents, multidimensional 3D functional contents using ultrasound.

This paper specifies multi-dimensional 3D image methods, technological characteristics of ${ }^{\bullet}$ multi-dimensional 3D images and the effect of ultrasound on humans, as noted especially in Chapter 2. Then, in Chapters 3, 4, and 5, changes in SMR waves were analyzed with the change in the levels of 3D feeling and sound stimulation. In the last chapter, Chapter 6 , the conclusion, and suggested measures for using the research results are presented $\lambda$,

\section{Related Works}

The basic principle of the 3D image can be referred to that which considered the disparity of two eyes, just like the human eyes. The distance between the human eyes is $65 \mathrm{~mm}$. Likewise, the left and right eyes are different retinas. Human beings perceive form multidimensionally while the brain interprets this fine difference. At this time, the difference of the form that the retinas of the two eyes detect, is ealled the disparity of two eyes. This can be considered the most important elementffoffeeling the three-dimensional effect [9].

The core of the three-dimensional dffect provided by the disparity of two eyes can be considered as the sense of depth or sense of distance. In other words, the brain perceives 2D form, perceived by each of the eyes according to the disparity of two eyes as multidimensional image. Through this perception process, human beings gain perspective and presence of the objects and images. Indeed, three-dimensional effect is what expresses sense of depth, sense of distance, perspective and others in a comprehensive manner, and high three-dimensional effect plays the role of increasing the sense of reality when it comes to image [9].

\subsection{Effects of the Sound of Utrasonic Waves on the Human Body}

It has been known since the 1920's that ultrasonic waves induce structural changes in biological tissue. Ultrasonic waves refer to sound waves in bandwidths that cannot be heard by human being; requencies higher than $20 \mathrm{kHz}$. However, ultrasonic waves, although they cannot be heard directly by human beings, can be perceived. The representative ultrasonic wave peception method is based on the vibration of sound waves passing through the cranial bone vias ayditory stimulation. Human beings perceive this due to the Labyrinth stimulation caused by cranial bone vibration. This phenomenon is called osteophony, and it is used for the treatment of tinnitus and other conditions [8]. Moreover, there is research that claims that ultrasonic waves do not merely affect the treatment of tinnitus, but that they also affect human brain vitalization. To measure the correlation between the sound of the ultrasonic waves and human beings' physiological reactions, an experiment has been conducted which has utilized ultrasonic waves and audible frequency bandwidth sound [9]. This experiment used four sound patterns; the sound of the ultrasonic waves (Low Cut Sound), audible frequency bandwidth sound (High Cut Sound), sound that mixed together ultrasonic waves and audible 
frequency bandwidth sound (Full Range Sound) and Baseline Sound (no sound) to stimulate the experiment's subjects. At the same time, EEG analysis and PET (positron emission tomography) filming were carried out to attempt to measure experiment subjects' states [9].

The experiment demonstrated that the alpha wave vitalization state was the highest when subjects were administered EEG analysis with the ultrasonic waves sound and audible frequency bandwidth sound were presented to subjects simultaneously. PET analysis demonstrated that the value of RCBF(regional cerebral blood flow) increased. When these analysis results are factored in, it is possible to assess that subjects do not perceive the sound of ultrasonic waves. However, indirect perception takes place in subjects' cerebrums, which induces the change in the brain's vitalization state

\section{User-Perceived 3D Feeling Analysis Following Location and Distance}

\subsection{Environmental Factors}

To analyze 3D Depth level of images, distance and location that will enabłe large number of viewers of the multi-dimensional images to view them effectively this researeh conducted the experiment in a university's lecture hall, targeting large number of people, and external factors (light, noise, etc.) were minimized to increase the engagement level for the image viewing. To increase the reliability level of the experiment, distance was measured for each of the locations where 3D images were viewed, targetinglecture halland subjects.

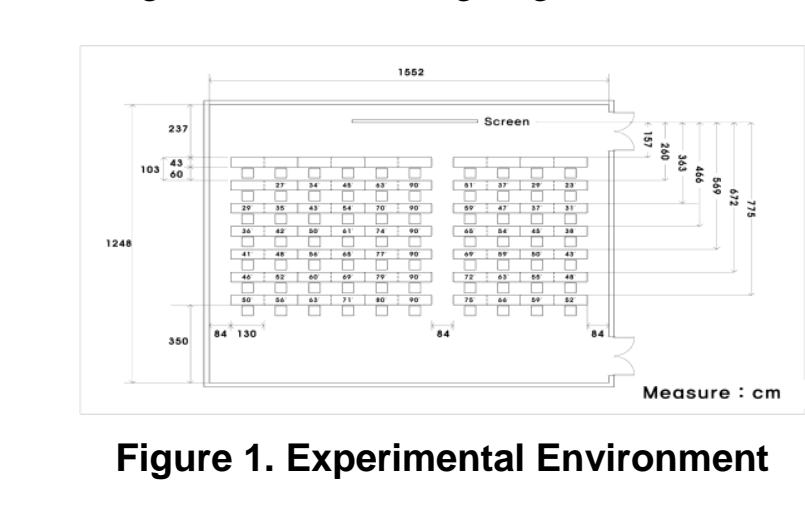

Overall experiment enviromment is shown on Figure 1. Figure 2 shows the users wearing Anaglyph eyeglasses for the $3 \mathrm{D}$ image viewing prior to the 3D Depth analysis experiment conducted to study users perception following the change in 3D Depth.
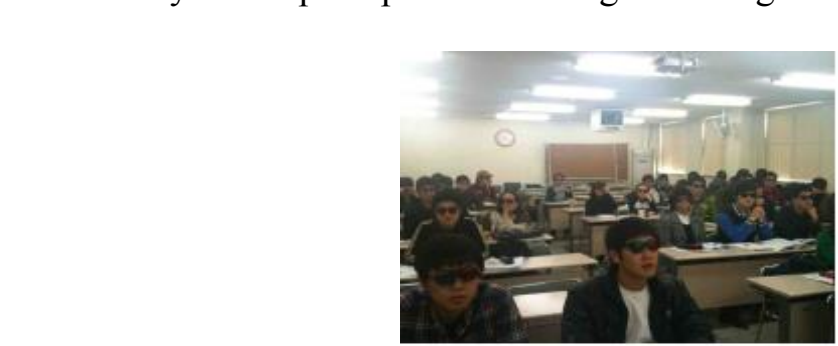

Figure 2. View of the users wearing Anaglyph eyeglasses prior to conducting the experiment 


\subsection{Methods}

Figure 3 is a capture of parts of the image used for the 3D Depth analysis experiment conducted to study users' perception following the change in $3 \mathrm{D}$ Depth. The images of the same scenes from (a) to (d) were changed depending on Depth level to change threedimensional effect. Subjects were then made to view them. Images were changed by increasing by 0.05 level from level 0 to level 1 to transform the 3D Depth. In case of level 0 , it manifests the 2D form image devoid of three-dimensional effect. In case of level 1 , it signifies the image with the image that will appear to the left eye and the right eye and that which was separated completely. Moreover, each of the images that appear on the left eye and the right eye get separated by $5 \%$ from the complete several overlaps when 3D Depth increases by 0.05 level. As such, images are shown to the subjects through the REd-Cyan eyeglasses.

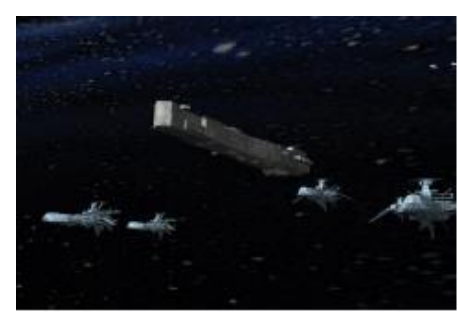

(a) Depth level 0

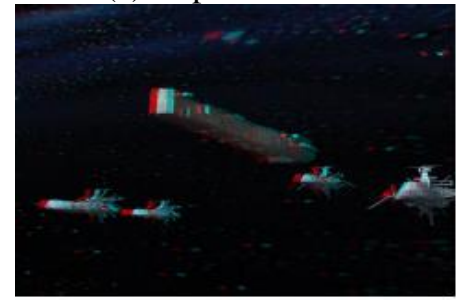

(c) Depthlevel 0.6

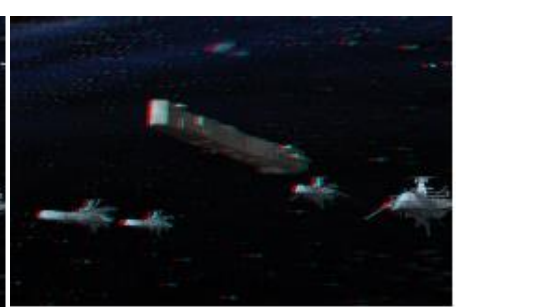

(b) Depth level0.3

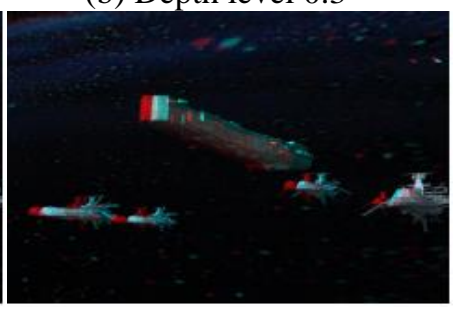

(d) Depth level 0.9

Figure 3. 3D images used for the experiment

In this paper, we conduct experiments as following steps.

\section{$\square$ Step 1}

While preparing for the 3D image to use during the experiment, each of the subjects were handed a data sheet where they can record the Anaglyph eyeglasses and feel for the threedimensional effeet. Then, they were asked to enter into the premise so that they could view the $3 \mathrm{D}$ image.

\section{$\square$ Step 2}

Subjects were shown image of the 3D Depth level 0 for one minute. When the image ended, subjects were asked to record on the data sheet whether they felt the three-dimensional effect.

$\square$ Step3

For the sake of the next stage experiment, subjects were asked to rest for one minute to minimize eye fatigue. 


\section{$\square$ Step 4}

This stage was conducted the same way as Stage 2 and Stage 3. When image ended, subjects were asked to view the 3D image with 3D Depth level increased by 0.05 once again for one minute. Then, they were asked to rest for one minute and to continue to repeat the process until the level turned to level 1 . To analyze 3D three-dimensional effect following subjects' distance and location, entire experiment process was repeated while the subjects' location was re-placed.

\section{Functional Games Benefit Optimization Technique Using Ultrasonic Waves' Auxiliary Sound}

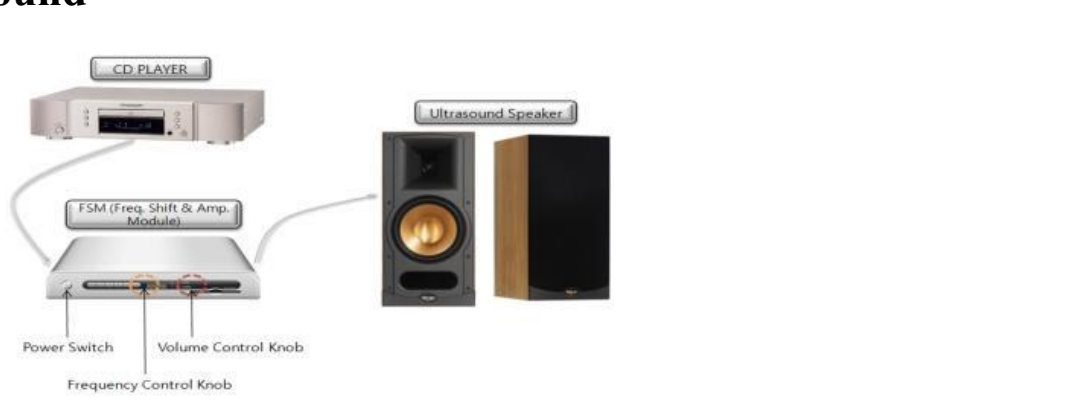

Figure 4. Overall USS (Ultrasound Speaker System) configuration

To study functional optimization measures tsing ultrasonic waves' auxiliary sound, this paper composed the USS (Ultrasound Speaker Systemx shown on Figure 4. USS (Ultrasound Speaker System) shifted frequency with ultrasonic waves at the FSM (Frequency Shift Module) when it comes to the audible frequency bandwidth sound effects played on CD players and used it as auxiliary sound. To ensure stable experiment subject stimulation, amplification and playing were carried out to stimulate the experiment subjects along with the sound effects of the audible frequency bandwidth. Auxiliary sound used on experiment subjects to stimulate then should be limited to the frequency bandwidth that is mostly above $20 \mathrm{kHz}$ and below $40 \mathrm{kHz}$ within the ultrasonic wave bandwidth.

\subsubsection{Frequency Shift Operation}

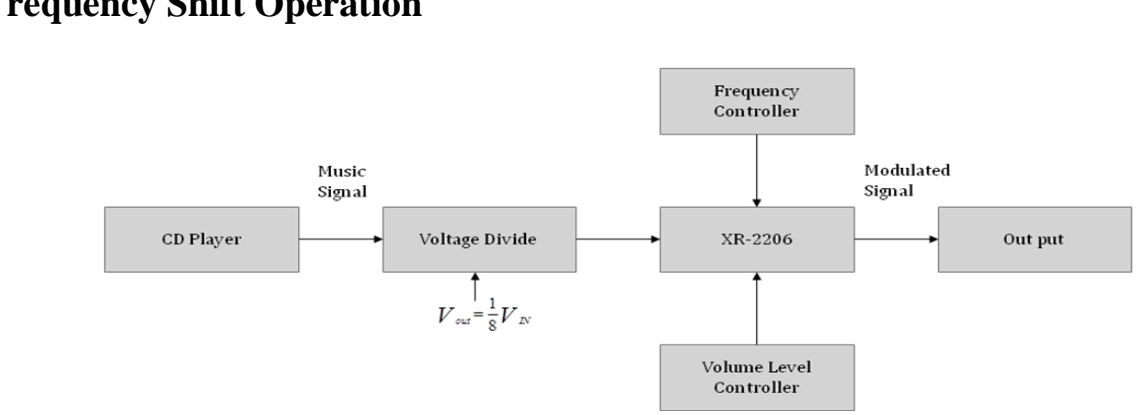

Figure 5. Frequency Shift Block Diagram

Figure 5 demonstrates the block diagram for the frequency shift part concerning $\mathrm{USS}^{8} V_{P-F} 1 V_{P-F} \frac{1}{8}$ (Ultrasound Speaker System). Frequency shift is mainly comprised of a music signal source, voltage division, frequency modulation IC and output. In the case of 
Figure 5, the music signal source receives the signal that is output from a $\mathrm{CD}$ player. Although the CD player's signal amplitude is output as Ro, the maximum output should be $\mathrm{Ga}$ in the case of XR-2206 (frequency modulation IC). Thus, it is necessary to reduce to Ro by distributing the voltage of the music signal. Then, it is necessary to input into XR-2206 and to adjust the frequency that will be shifted to the frequency adjustment controller from the XR-2206 circuit. The volume level controller is used to adjust the output amplitude to output ultrasonic waves. Figure 6 demonstrates a photo of the PCB concerning the FSM (Frequency Shift Module) produced to be used by this paper. Moreover, Figure 7 demonstrates the ultrasound speaker's frequency response characteristics graph used in this paper.

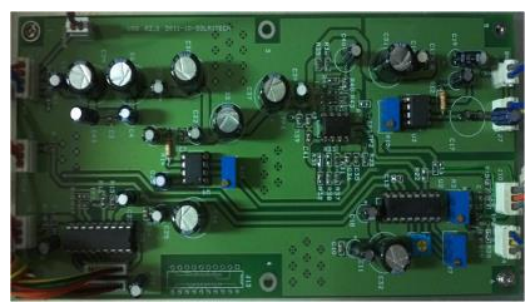

Figure 6. FSM (Frequency Shift Module) PCB photo

\subsection{Experiment Environment and Method}

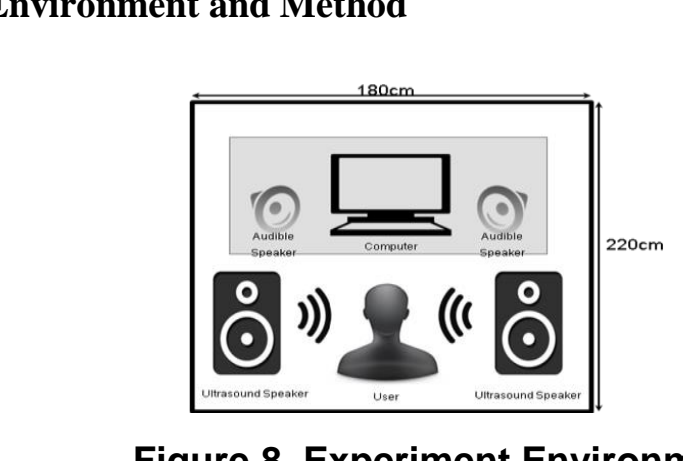

Figure 8. Experiment Environment

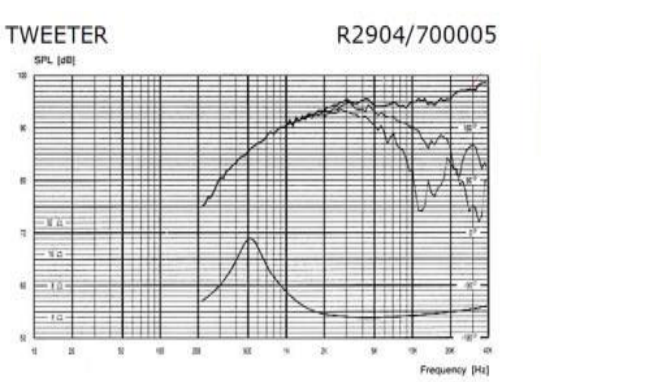

Figure 7. Ultrasound Speaker's Frequency Response Characteristics
Graph

To study the óptimization technique for functional games (functional games for concentration level increase) benefits using ultrasonic waves' auxiliary sound effects, this paper carried out an experiment using audible frequency bandwidth sound effects and ultrasonic wayes auxiliary sound effects when experiment subjects used 2D contents and 3D contents. The subjects focused on the functional game one person at a time (approximately $180 \times 220 \mathrm{~cm}$ ) depicted in Figure 8 . To increase the level of concentration and immersion into the game, the viewing distance was maintained at approximately $50 \mathrm{~cm}$ while outside elements (light, noise and others) were minimized. The experiment was conducted targeting a total of 30 men and women ( 15 men and 15 women). In the case of ultrasonic speakers, the distance for the dissemination of ultrasonic waves in the air was installed $30 \mathrm{~cm}$ away from the two sides of the user. At this time, the CD player used sounds of nature (for example, the sound of a stream) that helped relax the users, and increase their ability to concentrate. This sound was input as FSM, and got converted to $20 \mathrm{kHz}$ bandwidth ultrasonic waves' auxiliary 
sound. While carrying out the experiment, subjects got stimulated by the audible frequency bandwidth sound effect, the audible frequency bandwidth sound effect and ultrasonic waves' auxiliary sound effect at the same time. At this time, the personnel responsible for conducting the experiment, gathered data on the experiment subjects' brain waves to analyze immersion and concentration levels. This paper used the relative energy value of the SMR waves when it came to the index related to immersion and concentration levels.

\subsubsection{Experiment Methods}

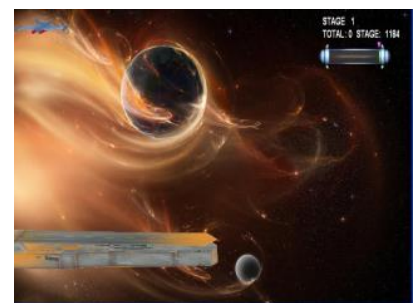

(a)2D Functional Contents

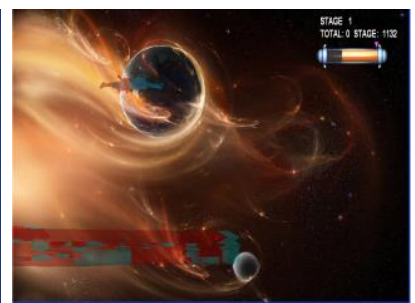

(b) 3D Functional Contents

\section{Figure 9. 2D and Multi-Dimensional 3D Form s Functional Game}

Figure 9 demonstrates the functional game used onthis paper (Anaglyph method). A multidimensional 3D form was produced first and foremost in order to study the measurements to optimize the benefits of the functional games of $/ 2 \mathrm{D}$-and multi-dimensional 3D formats. Then, the results were converted into $2 \mathrm{D}$ functional games. The benefits of the functional games of the 2D and 3D method were compared and analyzed.

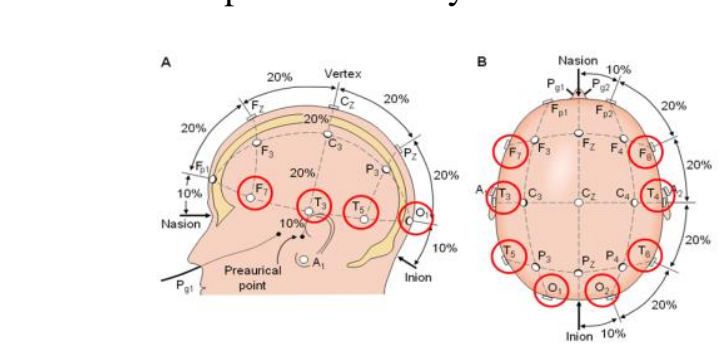

Figure 10. 10-20 Eterorfe Displacement Diagram Based on the International Standard Eterorfe Displacement

Figure 10 demonstrates the eterorfe displacement location used for the measurement of brain waves used in this experiment. As for the displacement location, it was selected according to 10-20 international standard. The location of the cerebrum related to concentration and immersion levels was considered to select eight locations; F7, F8, T3, T4, $\mathrm{T} 5, \mathrm{~T} 6, \mathrm{O}$ and $\mathrm{O} 2$ (256Hz Sampling rate, $12 \mathrm{bit} \mathrm{A} / \mathrm{D}$ conversion).

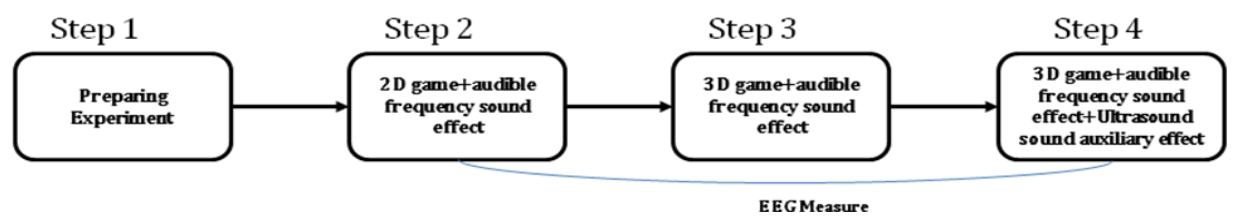

Figure 11. Experiment Method 


\section{$\square$ Step 1}

A disk electrode for the measurement of brain waves was attached at the "F7, F8, T3, T4, T5, T6, O1, O2" locations on the experiment subjects' heads based on Figure 10's 10-20 international standard eterorfe displacement, and 2D functional games, and 3D multidimensional functional games, audible frequency bandwidth sound effects and ultrasonic waves' auxiliary sound effects and others that are to be used in the experiment were prepared.

$\square$ Step 2

Experiment subjects were shown 2D functional games, as proposed in Figure 9(a). At this time, audible frequency bandwidth sound effects were presented to the experiment subjects. The experiment supervisor measured subjects' brain waves for a period of five minutes while they were playing 2D functional games.

$\square$ Step 3

Experiment subjects who wore blue-red glasses for viewing multi-dimensional 3D images (Anaglyph method) played 3D functional games. At this time, the audible frequency bandwidth sound effects that were used during Step 1-was presented to the experiment subjects. Moreover, the supervisor measured experinentsubjects'brain waves for a period of five minutes while the subjects were engaged in the games, the same length of time as during the $2 \mathrm{D}$ game.

$\square$ Step 4

Experiment subjects played functionar games under the same environment as that of Step 3. At this time, audible frequency bandwidth sound effects and ultrasonic waves' auxiliary sound effects were presented to the subjects at the same time, and the supervisor measured their brain waves for five minutes.

\section{Experiment Results}

\subsection{Analysis of the time when the feel for the three-dimensional effect started}

Table 1 shows the results on the analysis of the time when the three-dimensional effect was felt following the distance and location of the subjects tested for this research. Table (1) (for example, 0.3) shows the level of separation of the images that will be shown to the left eye and the right eye by factoring in the disparity of two eyes. In case of 0 the two images are overlapped completely. In case of 1 , the two images were separated completely. 


\section{Table 1. Analysis of the time when the users start to feel the three-dimensional effect by distance and location}

\begin{tabular}{|c|c|c|c|c|c|c|c|c|c|c|c|c|c|}
\hline & Column 1 & Column 2 & Column 3 & Column 4 & Column 5 & Column 6 & Column 7 & Column 8 & Column 9 & Average & $\begin{array}{c}\text { Total } \\
\text { av erage }\end{array}$ & Rank & Proportion \\
\hline \multirow{2}{*}{ Row 1} & & & & 0.2 & 0.05 & 0.2 & 0.45 & $\bar{x}$ & 0.6 & 0.3 & \multirow[b]{2}{*}{0.2} & \multirow{2}{*}{2} & \multirow{2}{*}{$9 / 10$} \\
\hline & & & & & 0.15 & 0.15 & 0.1 & 0 & & 0.1 & & & \\
\hline \multirow{2}{*}{ Row 2} & & 0.45 & & 0.3 & 0.25 & & & 0.25 & & 0.31 & \multirow{2}{*}{0.18} & \multirow{2}{*}{1} & \multirow{2}{*}{$5 / 6$} \\
\hline & & & & $\mathrm{x}$ & 0.05 & & & & & 0.05 & & & \\
\hline \multirow{2}{*}{ Row 3} & & & 0.3 & 0.05 & & 0.25 & & 0.05 & $\mathrm{x}$ & 0.16 & \multirow{2}{*}{0.25} & \multirow{2}{*}{4} & \multirow{2}{*}{$5 / 7$} \\
\hline & & & & $\mathrm{x}$ & & & & 0.35 & & 0.35 & & & \\
\hline \multirow{2}{*}{ Row 4} & 0.65 & 0.25 & & 0.15 & 0.1 & & 0.3 & 0.25 & 0.15 & 0.26 & \multirow{2}{*}{0.29} & \multirow{2}{*}{5} & \multirow{2}{*}{$10 / 12$} \\
\hline & & 0.15 & & $\mathrm{x}$ & 0.5 & & 0.3 & $\mathrm{x}$ & & 0.31 & & & \\
\hline \multirow{2}{*}{ Row 5} & & & & 0.05 & 0.1 & $\mathrm{x}$ & & 0.45 & $\mathrm{x}$ & 0.2 & \multirow{2}{*}{0.2} & \multirow{2}{*}{2} & \multirow{2}{*}{$4 / 9$} \\
\hline & & & & & 0.2 & $\mathrm{x}$ & & $\mathrm{x}$ & & 0.2 & & & \\
\hline \multirow{2}{*}{ Row 6} & 0.8 & $\mathrm{x}$ & 0.15 & 0.05 & & & 0.25 & 0.15 & 0.25 & 0.27 & \multirow{2}{*}{0.48} & \multirow{2}{*}{6} & \multirow{2}{*}{$7 / 10$} \\
\hline & & 0.8 & $\mathrm{x}$ & & & & 0.6 & & $\mathrm{x}$ & 0.7 & & & \\
\hline \multirow{2}{*}{ Row 7} & 1 & $\mathrm{x}$ & 0.45 & 0.2 & & 0.05 & 0.05 & 0.25 & & 0.33 & \multirow{2}{*}{0.61} & \multirow{2}{*}{7} & \\
\hline & & $\mathrm{x}$ & $\mathrm{x}$ & & & $\mathrm{x}$ & 0.9 & $\mathrm{x}$ & & 0.9 & & & \\
\hline $\begin{array}{c}1^{\text {st }} \\
\text { average }\end{array}$ & 0.81 & 0.35 & 0.3 & 0.14 & 0.12 & 0.16 & 0.25 & 0.23 & 0.33 & & & & \\
\hline $\begin{array}{c}2^{\text {If }} \\
\text { average }\end{array}$ & $\mathrm{x}$ & 0.47 & $\mathrm{x}$ & $\mathrm{x}$ & 0.22 & 0.15 & 0.47 & 0.17 & $\mathrm{x}$ & & & & \\
\hline $\begin{array}{c}\text { Total } \\
\text { average }\end{array}$ & 0.81 & 0.41 & 0.3 & 0.14 & 0.17 & 0.15 & 0.36 & 0.2 & 0.331 & & & & \\
\hline Rank & 9 & 8 & 5 & 1 & 3 & 2 & 7 & 4 & & & & & \\
\hline Phoportion & $3 / 3$ & $4 / 7$ & $3 / 5$ & $7 / 10$ & $8 / 8$ & $4 / 7$ & $8 / 8$ & $8 / 12$ & $3 \times \sqrt{6}$ & & & & \\
\hline
\end{tabular}

According to Table 1, experiment environment of the Figure 1 demonstrates that the 3D Depth level is low when it comes to the feel for the three-dimensional effect when users are closer to the screen. In terms of the distance, the effect is optimal while users are at the Row 1 and Row 2. Moreover, 3D Depth level was low when it comes to the feel for the threedimensional effect when users were situated at the center of the screen. In terms of the location, the order was Column 4, Colunn 5 and Column 8. Moreover, the analysis showed that the average 3D Depth level from which subjects start to feel the three-dimensional effect is 0.28 level.

\subsection{Analysis of the time when users stop feeling the three-dimensional effect}

Table 2 shows the analysis on the time when the subjects stop to feel the three-dimensional effect following distance and location. Table 2 (for example, 0.3 ) factored in the disparity of two eyes to demonstrate the level of separation of the images to be shown separately to the left and right eyes. In case of 0.0 the two images were overlapped completely. In case of 1 , the two images were separated completely. The analysis shows that the three-dimensional effect is felt continually eyen when the level of separation of the two images increases when users move to the center of the left and right sides from the center of the screen. In case of the center seat, it is possible to know that the users feel as the separated two images instead of feeling the images as multi-dimensional images when the level of separation for the two images increase. The distribution in terms of the location when it comes to the results of this experiment conducted targeting subjects in groups produced this order; Row 1, Row 3 and Column 8, which demonstrate that the separation level (3D Depth level) of the image that ends when it comes to the feel for the three-dimensional effect is high. Analysis following distance when it comes to the separation level (3D Depth level) of the image when the feel for the three-dimensional effect ends was low in this order; Column 4, Row 2 and Row 1. 
Table 2. Analysis of the time when the feel for the three-dimensional effect ends following distance and location

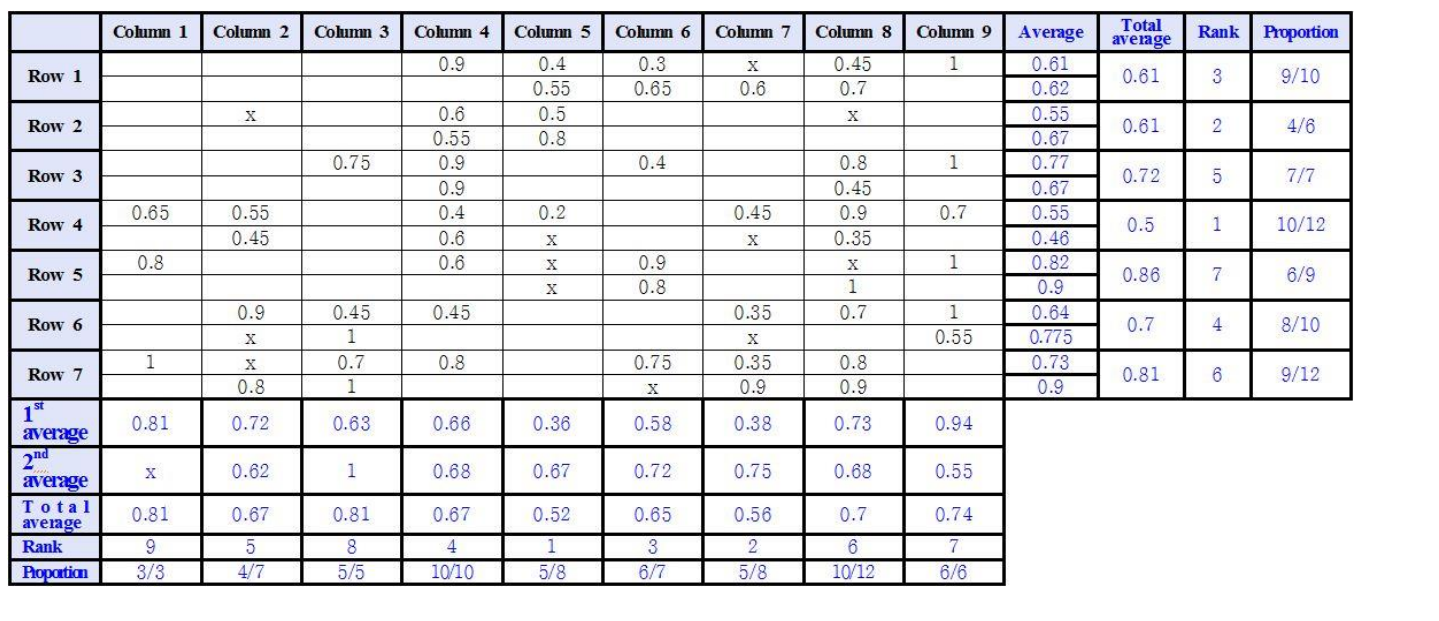

\subsection{Analysis of functional game's benefits (concentration and immersion levels)} following visual and hearing stimulation

To carry out an analysis of functional games' benefits following the changes in the visual and hearing stimulation executed by this paper, some $(30$ frames) of the change in the SMR index value by each channel following experiment subjects' eterorfe displacement location and type of visual and hearing stimulation of the Table 3 were manifested, and these were shown in Figure 12 as a graph.

Table 3. Analysis of Functional Games'Benefits (relative value of SMR index) Following Visual and Hearing Stimulation

\begin{tabular}{|c|c|c|c|c|c|c|c|c|c|c|c|}
\hline \multicolumn{3}{|c|}{$1 \mathrm{ch}(\mathrm{F} 7)$} & & & & \multicolumn{3}{|c|}{$3 \operatorname{ch}(\mathrm{T} 3)$} & \multicolumn{3}{|c|}{$4 \operatorname{ch}(\mathrm{T} 4)$} \\
\hline $\begin{array}{c}\text { Step 2 } \\
\text { (Fig 11) }\end{array}$ & $\begin{array}{r}\text { Step 3 } \\
\text { (Fig 11) }\end{array}$ & $\begin{array}{r}\text { Step 4 } \\
\text { (Fig 11) }\end{array}$ & & $\begin{array}{l}\text { Step } \\
\text { (Fig } 1\end{array}$ & (Fig 11) & $\begin{array}{c}\text { Step 2 } \\
\text { (Fig 11) }\end{array}$ & $\begin{array}{c}\text { Step 3 } \\
\text { (Fig 11) }\end{array}$ & $\begin{array}{c}\text { Step 4 } \\
\text { (Fig 11) }\end{array}$ & $\begin{array}{c}\text { Step 2 } \\
\text { (Fig 11) }\end{array}$ & $\begin{array}{c}\text { Step 3 } \\
\text { (Fig 11) }\end{array}$ & $\begin{array}{r}\text { Step 2 } \\
\text { (Fig 11) }\end{array}$ \\
\hline .04499 & 0.039873 & 0.037468 & 0027009 & 0.02636 & 0.029083 & 0.016552 & 0.020247 & 0.019921 & 0.011316 & 0.013569 & \\
\hline 039908 & 554 & & 0.025121 & $00_{2}$ & 0.023046 & 0.019116 & 0.016296 & 0.019114 & 013658 & 0.013171 & 13661 \\
\hline 034009 & 0.032759 & .048186 & 0.019097 & 0.018327 & 0.027413 & .012247 & .018832 & 017921 & 0.011256 & .01131 & 013408 \\
\hline 037751 & 0.040093 & 0.033412 & & 0.02381 & 0.027939 & 0.014 & 0.01 & & 192 & 015 & \\
\hline 030732 & 0.040524 & .043272 & & 0.02007 & 0.023229 & 0.013424 & 0.01 & 17325 & 06 & 0.013122 & 994 \\
\hline 035829 & 0.035 & .04312 & & 0.023696 & 0.019951 & 0.013449 & 0.021764 & 0.014123 & 2219 & 0.011905 & 992 \\
\hline 031697 & 0.038191 & & & 0.027339 & 0.025742 & 0.012794 & 0.021018 & & & 0.012181 & \\
\hline & 0.036929 & & 0. & 0.0202 & 0.024745 & $0.010^{-}$ & 0.01 & & & & \\
\hline & 0.041746 & & 0.020223 & 0.0213 & 0.018778 & 0.014 & 0.0 & 22 & 61 & 11 & 406 \\
\hline 988 & 884 & 0.045953 & 0.020226 & 0.022079 & 0.018928 & 0.011864 & 0.01 & 2 & 923 & 16 & 842 \\
\hline 077 & 0.04 & 0.039478 & 0.0 & 0.024404 & 509 & 0.0 & 0. & & 69 & 94 & 63 \\
\hline 055 & 0.0 & 0.03 & 0.02 & 0.022618 & 274 & 88 & 64 & & 37 & & \\
\hline 048 & & 0.03 & 0.0 & 0.021751 & 565 & & & & & & \\
\hline & 0.0 & 0.037251 & 0.023152 & 0.02644 & 0.01837 & 0.014823 & 0.02 & & 37 & 46 & 311 \\
\hline & 329 & 0.022288 & 0.019374 & 0.026214 & 0.019766 & 0.015 & 0.02 & 0. & 56 & 58 & 0.016172 \\
\hline & 0.05 & 0.0 & 0.0 & 0.02 & 845 & 0.01 & & & & & \\
\hline & 0.051806 & 0.038 & 0.020083 & 0.0 & 0.027044 & 0.015 & 0.0 & & & & \\
\hline & 0.04 & 0.038688 & 0.0285 & & 0.024086 & 0.0216 & & & & & \\
\hline 014 & 0.04 & 0.05 & 0.021262 & 0.02 & 0.025897 & 0.01 & 0.0 & & 666 & 33 & \\
\hline & 0.04 & & & 0.0 & 113 & & & & & & \\
\hline & 0.0 & & & & 107 & & & & & & \\
\hline 323 & 0.05 & 0.0 & 0.0 & 0.0 & 574 & 24 & & & & & \\
\hline & 0.05 & 0.04 & 0.021559 & 0.032778 & 0.021298 & 0.01875 & 0.02 & & 14 & 99 & \\
\hline 051071 & 0.045723 & 0.04 & 0.02372 & 0.030068 & 0.025017 & 0.020883 & 0.02 & & 85 & 127 & 166 \\
\hline .051153 & 0.043059 & 0.043672 & 0.026932 & 0.022762 & 0.022168 & 0.019061 & 0.024888 & 0.022494 & 0.018618 & 7251 & \\
\hline 037 & 0.041741 & 0.046683 & 0.029894 & 0.024281 & 0.028484 & 0.017959 & 0.02395 & 0.023698 & 0.018475 & & 693 \\
\hline & 0.04 & 0.0 & 0.030962 & 0.025503 & 0.024319 & $0.018^{\prime}$ & 0.01 & & 401 & 507 & \\
\hline & 0.04 & 0.05 & 0.029833 & 0.024385 & 0.023804 & 0.018 & 0.0 & 0.0 & 227 & & \\
\hline 055676 & 0.043341 & 0.04611 & 0.026084 & 0.024491 & 0.02851 & 0.014281 & 0.02 & 0.02785 & 0.015538 & 0.015638 & 0.019931 \\
\hline
\end{tabular}




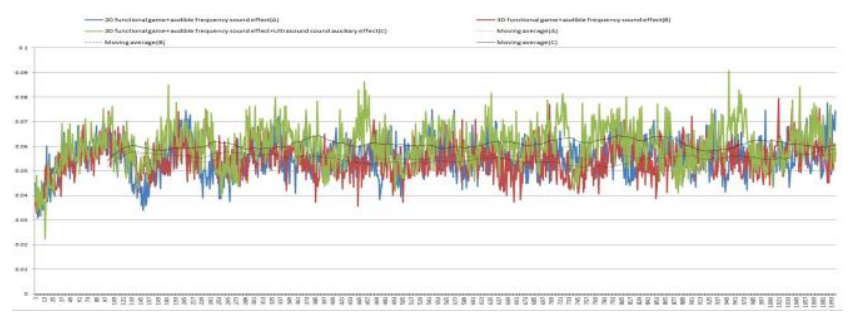

(a) $1 \mathrm{Ch}-\mathrm{F} 7$

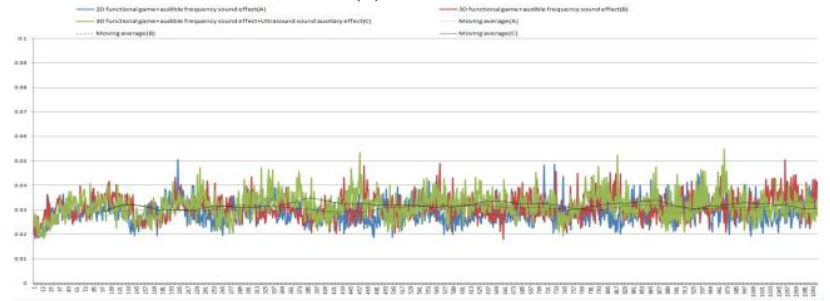

(b) $2 \mathrm{Ch}-\mathrm{F} 8$

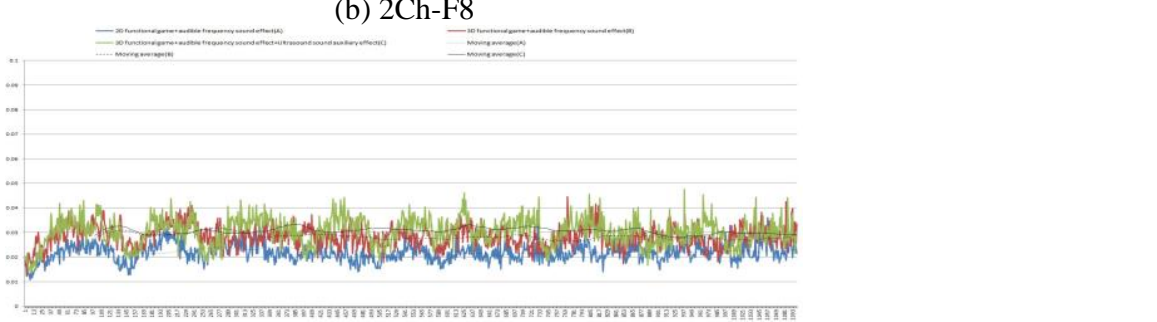

(c) $3 \mathrm{Ch}-\mathrm{T} 3$
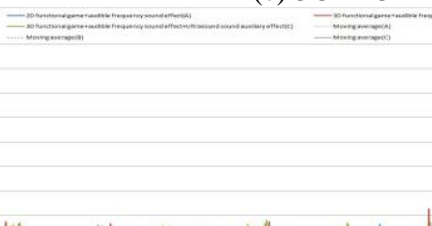

Y

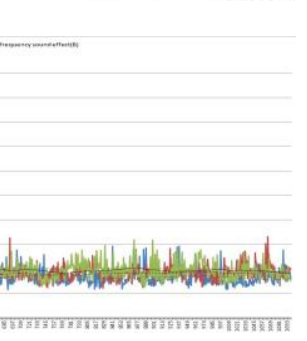

(d) $4 \mathrm{Ch}-\mathrm{T} 4$

\section{Figure 12. Analysis of Functional Games' Benefit (relative value of SMR index)} Graph Following Visual and Hearing Stimulation

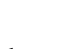

The experiment results showed that the relative energy value of the SMR wave bandwidth (12 16hz), which is the measure for showing the concentration level for all of the frontal lobe is high in the order of Step 4, Step 3 and Step 2. In some parts of the Ch1, there were some parts where the results of the Step 2 were higher than those of the Step 3 and Step 4, but the effect on the overall result was rather lacking.

\section{Conclusion}

To study measures for the optimization of functional games' benefits, this paper verified levels of perceived 3D feel (time when users started and finished experiencing this 3D feeling), audible frequency bandwidth sound effects and ultrasonic waves auxiliary sound effects following distance and location.

According to the results of the experiment executed in this paper, 3D feel's depth level that enables users to feel the 3D images most effectively and that manifested the highest immersion level was different depending on distance and location. To remove the effect of distance and location, the time when the users started to feel the 3D experience, with respect 
to distances and locations, was manifested as depth level 0.28. As for the time when the users stopped experiencing 3D effects, it was depth level 0.68. Details on the time when users started and stopped this feeling were shown in Table 4.

Figure 13 demonstrates the results of the analysis following functional games' feeling and sound effects, and auxiliary sound effects. According to Figure 13, it is possible to know that 3D multi-dimensional functional games are more effective when it comes to inducing concentration and immersion levels compared to 2D functional games.

In the case of 3D multi-dimensional functional games, the effect of inducing concentration and immersion levels was higher when audible frequency bandwidth sound effects and ultrasonic waves' auxiliary sound effects were used at the same time, compared to the instance in which only audible frequency bandwidth sound effects were used.

\section{Table 4. Distribution of the Time When Users Started and Stopped Feeling 3D} Effects Following Depth Level

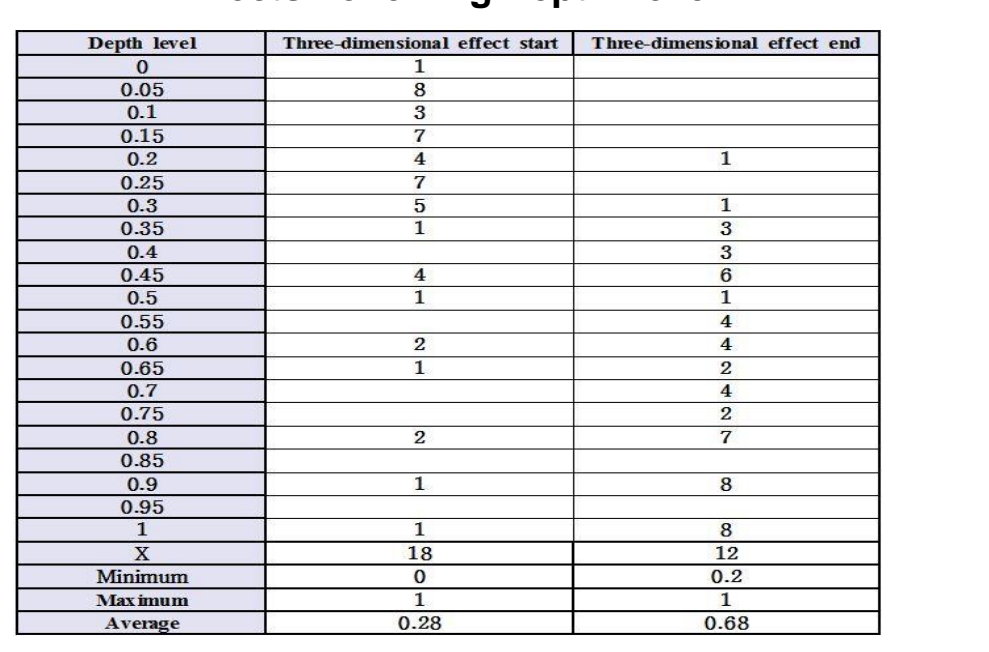

Figure 13's (a) demonstrated the resylts of the analysis conducting on the relative SMR wave energ among the chamiels following the change in visual and hearing stimulation. Meanwhile, (b) maniflested the results of the analysis for the absolute SMR wave energy among the channels following the change in the visual and hearing stimulation. When this is factored in, immersion and concentration levels improved by approximately $33.7 \%$ in the case of functional game contents that use audible frequency bandwidth sound effects and ultrasonic waves auxiliary sound effects of that use merely audible frequency bandwidth sound effects of the 2D form.

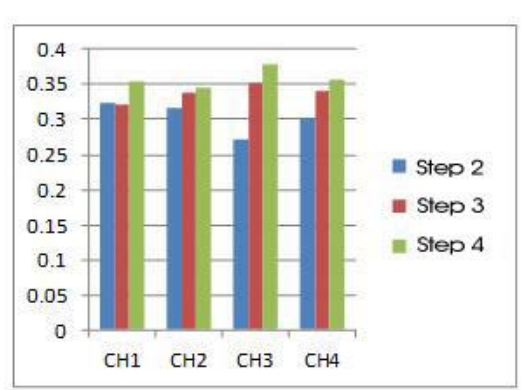

(a)Analysis of relative value among channels (SMR wave)

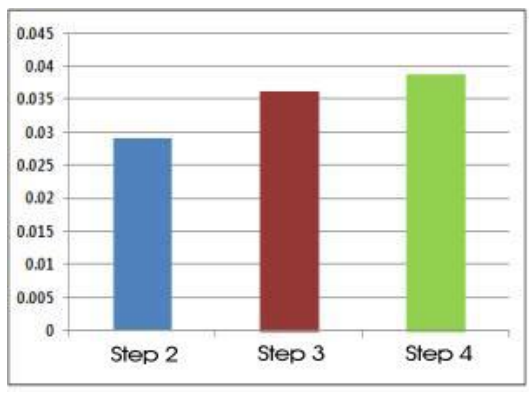

(b)Analysis of absolute values (SMR wave)

Figure 13. Results of the SMR Wave Analysis 
When the results of the research conducted by this paper are utilized, it is possible to use the results as a guideline for the production of 3D multi-dimensional functional game contents for the optimization of functional game benefits. Moreover, when users use 3D multi-dimensional functional games, it may be possible to select the distance and location that enables users to experience 3D effects more optimally. Moreover, it is judged that the results may be used in diverse areas to increase immersion and concentration levels of functional games using auxiliary sound effects.

\section{Acknowledgements}

This research was supported by Basic Science Research Program through the National Research Foundation of Korea(NRF) funded by the Ministry of Education, Science and Technology (NRF2012R1A1A4A01011858).

\section{References}

[1] I. Sexton and P. Surman, "Stereoscopic and auto-stereoscopic display systems", Signal Processing Magazine IEEE, vol. 16, (2002), pp. 85-99.

[2] D. M. Hoffman, A. R. Girshick, K. Akeley and M. S. Banks Vérgence-accommodation conflicts hinder visual performance and cause visual fatigue", Journal of Vision, vol. 8, (2008), pp 1-30.

[3] D. -h. Kim, S. -h. Choi and G. -h. Sohn, "Forecasting of the 3D Video's Fatigue Level that Factor in the Characteristics of the Viewers", The Korean Society of Broadoast Engineers journal, vol. 16, (2011), pp. 331338

[4] M. Lambooij, M. F. Fortuin, W. A. IJsselsteijn, B N.W. Evans and I. Heynderickx, "Measuring Visual Discomfort associated with 3D Displays", Journalof Sóciety of Information Displays, vol. 18, (2010), pp. 931-943.

[5] M. Halle, "Auto-stereoscopic displays and compluter graphic", Computer Graphics, ACM SIGGRAPH, vol. 31, (1997), pp. 58-62.

[6] J. Choi, D. Kim, B. Ham, S. Choi and K. Sohn, "Visulf fatigue evaluation and enhancement for 2D-plusdepth video", International Conference on Image Rrocessing, (2010), pp. 2981 - 2984.

[7] L. Zhang and W. J. Tam, "Stereoscopie image generation based on depth images for 3DTV", IEEE Trans. on Broadcasting., vol. 51, (2005), pp. 191-199.

[8] M. L. Lenhardt, "Ultrasonic Heaying in Humans: Applications for Tinnitus Treatment", Int Tinnitus J., vol. 9, (2003), pp. 69-75.

[9] Hiroshi, Shibasaki, Fuwamøto, N. Kawa1, T Maekawa, S. Nakamura, H. Fukuyama, T. Oohashi, E. Nishina, M. Honda, Y. Yonekura and Yoshitaka, "Inaudible High-Frequency Sounds Affect Brain Activity: Hypersonic Effect", AJP - JN Physiol., vol. 83, (2000), pp. 3548-3558.

[10] Korea Creative Contents Agency, “A Study on the Analysis of the US 3D Contents Industry Structure and on the Entry Measures", (2010) Degember 28.

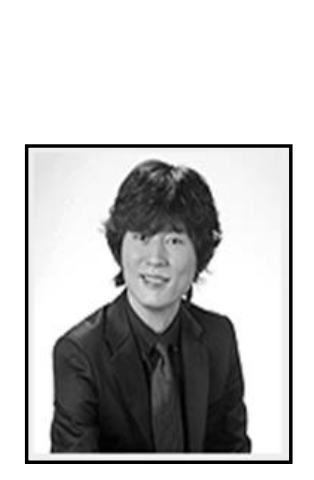

\section{Authors}

\section{Jeong-Hoon Shin}

Address: School of Computer \& Information Communication Engineering,Catholic University of Dae-Gu, 330 Geumrak 1-Ri HauangEup Gyeonsan-Si Gyeongbuk, Korea

Education \& Work experience:

Jeong-Hoon Shin received a B.S and M.S and Ph.D. in Electronics Engineering from Sungkyunkwan University, Korea in 1991, 1993 and 2005 . 
From 1994 to 2002, Mr. Shin was an engineer at DACOM R\&D Center, where he was involved in the design and performance analysis of wired and wireless communication network.

From 2003 to 2004, he was an extraordinary professor at Indukcollege, Seoul, Korea. Since March 2006, he has been a professor at Catholic University of Daegu, Kyungsan, Korea. His current research focuses on Brain Computer Interface, the five senses recognition and synthesis. He is a member of TTA, KDISS and ASK. And he is also a member of valuation committee in IITA.

Tel: +82-53-850-2748

E-mail: only4you@cu.ac.kr

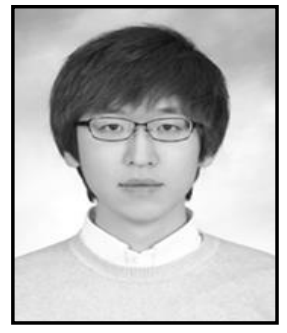

\section{Seong-Jun Lee}

Engineering, Catholic University of Dae-Cu, 330 Geumrak 1-Ri Hauang-Eup Gyeonsan-Si Gyeongbuk, Korea

Education \& Work experience:

2005. 3. Entrance into Computer \& Information Communication Eng. of Catholic University of Dae-Gu,

2013. 12.-Today. Schod of Information Technology Eng, Catholic University of Dae-Gu,HeI Lab.(Researcher)

Tel: +82-10-4529-6329

E-mail: 1114444@cu.ac.kr

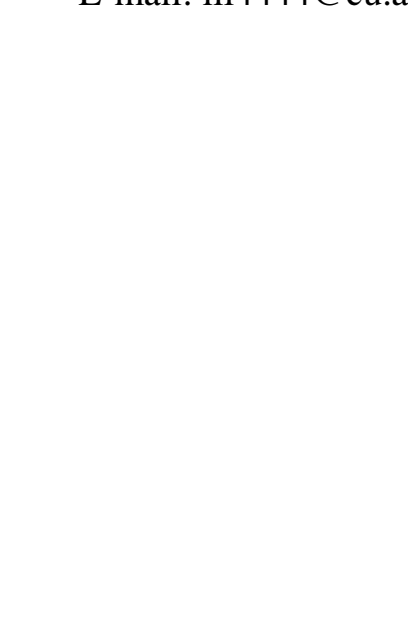

